\title{
Fungicidal and antistress properties of the preparation based on triterpene acids on spring barley
}

\author{
V.P. Korotky ${ }^{1}$, N.V. Smolin ${ }^{2}, A . N . \mathrm{Nikolsky}^{2}$, V.A. Ryzhov ${ }^{1, *}$, and I.V. Korotky ${ }^{1}$ \\ ${ }^{1}$ LLC "Chiminvest", Nizhny Novgorod, Russia \\ ${ }^{2}$ Mordovian State University, Saransk, Republic of Mordovia, Russia
}

\begin{abstract}
The most effective concentration range of the studied preparation synthesized on the basis of triterpene acids is in the range of $0.01-0.005 \%$. In the conditions of water-salt stress, the studied preparation increased laboratory germination of seeds, length of seedling and root of spring barley compared with the control variant. At a concentration of $0.01-0.005 \%$, the preparation reduced the intensity of the pathogenic complex development on the seed material of spring barley, but was significantly inferior in effectiveness to the synthetic protectant. At the same time, unlike a fungicide, the preparation did not have a depressing effect on the development of barley in the initial stages of ontogenesis.
\end{abstract}

\section{Introduction}

The use of growth regulators in agricultural production is aimed at reducing the harmful effects of pathogenic organisms, preventing lodging of grain crops and grain molting, increasing yield and improving the quality of grown products, accelerating maturation, improving fruit setability, facilitating mechanized harvesting [1].

The adaptogenic effect of preparations of this series is leveling the stress effects caused by a sharp change in growing conditions [6]. In the conditions of the Middle Volga region with an unstable climate (uneven precipitation, frequent droughts, recurrent and early frosts), plant productivity is often unpredictable. In many farms of this region, more than half of the arable land in the structure of sown areas is occupied by grain crops, in which seedings favorable conditions are created for the development and spread of harmful organisms. Therefore, for a comprehensive assessment of each growth regulator action, it is necessary to check its effect on the infestation of plants with pathogens. On the one hand, it is necessary to study and identify the mechanism and degree of influence of regulators on plants, on the other hand, comparing a new preparation with known fungicides, it is possible to identify the mechanism and spectrum of its action on a pathogenic complex or on the most common and harmful diseases.

Every year, the range of growth regulators with a phytoncidal effect is expanded [7, 3, $10,9]$. The most promising group of growth regulators is triterpenic acids. The main

\footnotetext{
${ }^{*}$ Corresponding author: woodnn@yandex.ru
} 
components of a mixture of pine straw triterpene acids are compounds with a modified lanostane carbon skeleton. In general, triterpene acids of needles are a rather complex mixture of similar pairs of geometric isomers that differ in the stereochemistry of the molecule side chain, the structure of the tetracyclic carbon backbone and a substituent at the $\mathrm{C}(3)$ atom (usually a keto or hydroxyl group). One compound with a methoxy group at this atom was found as a minor component among the triterpene acids of needles.

A number of abiotic and biotic factors act on cultivated plants in the process of their growth and development, the effect of which can be both positive and negative. A negative manifestation of factors on the plant is a state of stress.

Stress is defined as any external abiotic or biotic influence that limits the rate of photosynthesis and reduces the ability of a plant to convert energy into biomass.

Of the abiotic factors that negatively affect the development of spring grain crops, water and salt stress is of the greatest importance. Water stress, affecting plant growth, can vary in the degree of negative action, often combined with other stresses (high temperature, salt) [18]. Salt stress affects germination, growth, photosynthesis, protein and energy synthesis, lipid metabolism, causes an imbalance of nutrients, oxidative stress [17].

In this regard, in order to save the crop, it is noted that it is necessary to use growth regulators and substances like them in crop production, in particular, in conditions of soil salinization, water scarcity, the influence of low or high temperatures, the negative impact of excessive amounts of xenobiotics, including heavy metals (HM) on cultivated plants [2].

Currently, synthetic fungicides are the main means of combating fungal diseases in crop production. Although the use of synthetic fungicides benefits agriculture by increasing crop yields, their intensive annual use causes certain concerns as a result of possible environmental pollution, potential risk to animal and human health. Moreover, with the duration of the use of fungicides of the same chemical class has become less effective due to the appearance of the effect of resistance in fungal pathogens. Thus, biological control using growth regulators and plant metabolic products is considered as an alternative method of pathogen control $[15,14]$.

Derivatives of triterpene acids or steroid glycosides, widely distributed in various plants [19]. Triterpenoids have polyphilic properties, interact with both lipophilic and hydrophilic components, which often have cytotoxic and other biological activity, including fungistatic activity [13]. The main mechanism of antifungal activity of triterpenoids is due to their ability to form complexes with sterols in fungal membranes and cause loss of membrane integrity with the formation of transmembrane pores [16].

Currently, in the practice of crop production, a positive effect of a number of preparations containing triterpene compounds on the cultures has been established. Novosil stimulated the growth and activated the protective functions of plants under the influence of stress factors $[12,5]$. Snare increased the field germination of seeds, the number and height of productive stems, the length and number of spikelets, the ear grain content, the weight of grain; accelerated maturation and improved the grain quality indicators (vitreousity, raw gluten content) [4]. Verva activated the growth of plants, increased their resistance to extreme environmental influences [11].

At the same time, a comprehensive study of triterpene-type preparations to reduce abiotic stress and the development of pathogenic organisms has not been conducted. The purpose of the research was to study the effectiveness of a phytoncidal agent containing triterpenic acids in reducing the growth and development of fungal pathogens, the possibility of using a new phytoncidal agent for germination energy and laboratory germination of spring barley seeds. 


\section{Research methods}

The objects of research are spring barley plants (Triticum aestivum L.) of the Zazersky 85 variety. The studied preparation (pine resin pyrolysis product) was provided by LLC "Chiminvest". A fungicidal protectant (90 g/l difenoconazole $+45 \mathrm{~g} / 1$ tebuconazole) was used as a comparison preparation. The studied preparation was used as an aqueous solution in concentrations of $0.1,0.01,0.05$ and $0.001 \%(10,1,0.5$ and $0.1 \mathrm{ml} / \mathrm{l})$.

The plant seeds for the experiment were sterilized in $70 \%$ ethanol, then washed with distilled water. After exposure for 18 hours in solutions of distilled water (control) $0.1,0.01$ and $0.001 \%$ solutions of the studied preparation, the growth energy and germination in Petri dishes were determined. Seeds for establishing a stress response were germinated in rolls on moistened filter paper in a thermostat for 7 days at $+24{ }^{\circ} \mathrm{C}$. Germination in the first 48 hours was carried out on a sodium chloride solution $(2 \%)$, the control was water. The length, wet and dry mass of roots and shoots of plants were measured.

To assess the intensity of pathogen damage, a method was used to assess the pathogenicity of root rot pathogens when treating them with protectants [8]. Spring barley grains were soaked for half an hour of the day in a solution of the studied preparation (5 $\mathrm{ml} / 1$ of sterile water), and a fungicidal protectant ( $6 \mathrm{ml} / 1$ of sterile water). The consumption of the working solution is $30 \mathrm{ml} / 100 \mathrm{~g}$ of seeds. After a day, the seeds were laid out in the planters on moistened filter paper in four repetitions. After a week of thermostating at a temperature of $24^{\circ} \mathrm{C}$, the number of infected seedlings and the degree of damage to the seedlings were considered using a 4-point scale: 0 - a healthy seedling; 1 - focal necrosis of the root and seedling tissue; 2 - necrosis of about $50 \%$ of the root and seedling area; 3 complete death.

\section{Research results}

The determination of the growth energy showed that the studied preparation in a concentration of $0.1 \%$ significantly inhibited growth processes. In this case, the decrease was $17 \%$. At low concentrations of the studied preparation $-0.01 \%$ and $0.001 \%$, the energy was higher than in the control - by 5 and $3 \%$, respectively, but there were no significant differences between the variants (Table 1).

Table 1. Effect of the concentrations of the studied preparation on the germination energy and germination of spring barley seeds, $\%$.

\begin{tabular}{|c|c|c|}
\hline Experiment option & $\begin{array}{c}\text { Growth energy } \\
, \%\end{array}$ & $\begin{array}{c}\text { Laboratory } \\
\text { germination rate, } \%\end{array}$ \\
\hline Control & 63 & 92 \\
\hline $0.1 \%$ solution of terpenic acids & 46 & 69 \\
\hline $0.01 \%$ solution of terpenic acids & 68 & 93 \\
\hline $0.001 \%$ solution of terpenic acids & 65 & 92 \\
\hline $\mathrm{LSD}_{05}$ & 4 & 8 \\
\hline
\end{tabular}

Seed germination was determined on the seventh day of the experiment. The previously established trend has been repeated. The studied preparation at a concentration of $0.1 \%$ significantly reduced the indicator due to the high content of active substances in the solution. No significant differences were found between the control and the variants with a concentration of $0.01 \%$ and $0.001 \%$.

It was found that the effective concentration of the preparation solution is in the range of $0.01-0.001 \%$. The following laboratory tests were continued in this interval. 
Determination of the growth energy of spring barley seeds under salt stress conditions showed that in the variant without germination of seeds in $\mathrm{NaCl}$ solution this indicator was significantly higher than other variants (Table 2).

Table 2. Effect of water-salt stress on the growth energy of spring barley seeds, $\%$.

\begin{tabular}{|l|c|c|c|c|}
\hline Experiment option & $\begin{array}{c}\text { Growth } \\
\text { energy, } \\
\%\end{array}$ & $\begin{array}{c}\text { Laboratory } \\
\text { germination } \\
\text { rate, \% }\end{array}$ & $\begin{array}{c}\text { Length } \\
\text { of seedling, } \\
\mathrm{cm}\end{array}$ & $\begin{array}{c}\text { Length } \\
\text { of roots, } \\
\mathrm{cm}\end{array}$ \\
\hline Control (water) & 63 & 89 & 6.3 & 3.1 \\
Water + NaCl & 43 & 60 & 2.7 & 2.0 \\
$0.01 \%$ solution TA-t $+\mathrm{NaCl}$ & 53 & 67 & 2.8 & 2.2 \\
$0.005 \%$ solution TA-t $+\mathrm{NaCl}$ & 51 & 71 & 3.2 & 2.2 \\
$0.001 \%$ solution TA-t $+\mathrm{NaCl}$ & 45 & 64 & 3.3 & 2.1 \\
LSD 05 & 6 & 5 & 0.4 & 0.5 \\
\hline
\end{tabular}

The treatment of spring barley seeds with the studied preparation, despite the decrease in growth energy compared to the control, was higher than in the variant with germination of seeds in a salt solution. Thus, at a concentration of $0.01 \%$ of the preparation, the growth energy was $10 \%$ higher, at a concentration of $0.05 \%-8 \%$, at a concentration of $0.001 \%-$ $2 \%$ higher. A similar trend has continued in determining the laboratory germination rate of seeds.

Compared with the control, the germination rate of seeds germinated in water-salt solution decreased by $29 \%$. At the same time, the treatment of seeds with the studied preparation increased this indicator by $4-11 \%$. At the same time, the best options were preparation concentrations of 0.01-0.005\%.

The positive dynamics of the studied preparation was also noted for the growth of seedlings and roots in this laboratory experiment. The maximum length of seedlings was noted on the control variant. Germination of seeds in water-salt solution significantly reduced the studied indicator - by $3.6 \mathrm{~cm}$ on average according to the experiment.

The use of the experimental preparation slightly increased the length of the seedlings compared to the water $+\mathrm{NaCl}$ variant. Thus, at the preparation concentration of $0.01 \%$, the increase in the length of the seedling was $0.1 \mathrm{~cm}$ at a concentration of $0.005 \%-$ by $0.5 \mathrm{~cm}$, at a concentration of $0.001 \%-0.6 \mathrm{~cm}$.

The water-salt solution also had a depressing effect on the growth of the roots of barley seedlings. If in the control variant this indicator averaged $3.1 \mathrm{~cm}$, then in the experimental variants the decrease was $25-31 \%$.

At the same time, the studied preparation increased the growth of the root compared to the variant where it was not used. The increase in this parameter was statistically unreliable, but it was recorded for all experiment repetitions.

Thus, the conducted studies indicate a decrease in the negative effect of water-salt stress on spring barley plants when using the preparation in concentrations of $0.01-0.001 \%$.

To determine the effect of the studied preparation on the intensity of seed damage by fungal pathogens, barley seeds with a high initial pathogenic index were selected (Table 3).

According to laboratory studies, the total degree of grain infection with pathogenic microorganisms was higher than the average $(22 \%)$. The suppression and death of seedlings were caused by fungi of the genus Fusarium spp., Bipolaris sorokiniana (Sacc.), Pyrenophora teres (Sacc.). At the same time, the fusarium-helminthosporiosis type of infection prevailed.

In the control variant, after 7 days of seed germination, a high degree of pathogen complex spread was established - more than $60 \%$. The use of a fungicidal protectant significantly reduced this indicator - by $58 \%$. Although the studied preparation was inferior to the synthetic protectant in its action, significant differences with the control were 
noted at all the studied concentrations. The decrease in the spread of pathogenic microorganisms was $20-22 \%$.

Table 3. The effect of the studied preparation on the spread of pathogen complex and the growth processes of spring barley seeds.

\begin{tabular}{|l|c|c|c|}
\hline Variant & $\begin{array}{c}\text { Complex } \\
\text { of pathogens, } \\
\%\end{array}$ & $\begin{array}{c}\text { Length } \\
\text { of seedling, cm }\end{array}$ & $\begin{array}{c}\text { Length } \\
\text { of roots, cm }\end{array}$ \\
\hline Control (dist. water) & 63 & 12.6 & 7.2 \\
Fungicidal protectant & 5 & 10.4 & 5.4 \\
0.01\% solution of terpenic & 43 & 16.2 & 9.9 \\
0.005\% solution of terpenic & 41 & 16.5 & 10.6 \\
LSD $05^{2}$ & 11 & 1.1 & 1.5 \\
\hline
\end{tabular}

An important parameter in determining the effect of preparation on the pathogen complex is the degree of disease development, since it determines the effect of diseases on the further growth and development of seedlings. The data obtained by us indicate a significant lesion of experimental plants in the control - more than $50 \%$ of necrosis.

Treatment of synthetic seeds with a fungicidal protectant significantly reduced the development of diseases. Mild injuries were noted in $20 \%$ of the studied plants, and their damage score was 1.9 lower compared to the control. The treatment of seeds with the studied preparation reduced the intensity of the lesion by $30-35 \%$ compared to the control, but also significantly inferior to the option with the use of the protectant based on synthetic chemical compounds.

It should be noted that the use of fungicidal protectants, despite its high effectiveness in combating a complex of pathogens, negatively affects the growth of seedlings and seed roots. The reduction in the seedling length compared to the control was $2.6 \mathrm{~cm}$. At the same time, the use of the studied preparation, together with a decrease in the lesion of barley plants, had a positive effect on increasing the seedling length. Compared with the control, this parameter increased by $3.6-3.9 \mathrm{~cm}$.

A similar trend was established when analyzing the length of the roots in spring barley. The fungicidal protectant slightly reduced this indicator - by $1.8 \mathrm{~cm}$ on average according to the experiment. The studied preparation consistently increased the root length compared to the control - on the variant with a concentration of $0.01 \%$ - by $2.7 \mathrm{~cm}$, on the variant with a concentration of $0.005 \%-3.4 \mathrm{~cm}$.

The conducted studies indicate the possibility of using the studied preparation for the vegetation of spring grain crops in a concentration of $0.01-0.005 \%$ to reduce the negative impact of abiotic factors and to increase resistance to a complex of pathogens.

\section{Conclusion}

The conducted studies allowed to establish the most effective range of concentrations of the studied preparation - 0.01-0.005\%. Under conditions of water-salt stress, the studied preparation significantly increases the laboratory germination of seeds, the length of the seedling and root of spring barley compared to the control variant.

At a concentration of $0.01-0.005 \%$, the preparation reduces the intensity of the development of spring barley pathogens, but is significantly inferior in effectiveness to the synthetic protectant. At the same time, the preparation does not have a depressing effect on the development of barley seedlings, unlike the fungicide. 


\section{References}

1. V.V. Vakulenko, Protection and quarantine of plants 12, 24-26 (2007)

2. T.S. Kolmykova, A.S. Lukatkin, Agrochemistry 1, 83-94 (2012)

3. V.N. Lazarev, E.V. Kovalenko, N.A. Kudryavtsev, Protection and quarantine of plants 9, 36-38 (2016)

4. G.I. Larionov, T.M. Zorkina, S.V. Kulemina, L.L. Martynovich, Agrochemistry 8, 5760 (2003)

5. G.L. Matevosyan, A.D. Shishov, A.S. Sadovnikov, Bulleting of the NovSU 86 (1), 116-119 (2015)

6. V.I. Morozov, A.I. Morozov, G.P. Pushkina, Protection and quarantine of plants 1, 30 (2005)

7. Z.G. Pozharsky, I.N. Bokanga, Protection and quarantine of plants 5, 34-35 (2016)

8. M.A. Revkova, Agrobiological justification of the protection of spring barley from the main pathogens of root rot in the CCR, 22 (S.-Pb.-Pushkin: VIZR, 2011)

9. T.A. Ryabchinskaya, T.V. Zimina, Agrochemistry 12, 62-92 (2017)

10. A.M. Sorokin, Chief agronomist 1, 12-15 (2017)

11. T.V. A.V. Khurshkainen, Kuchin, News of the Komi Scientific Center of the Ural Branch of the Russian Academy of Sciences 1 (5), 17-23 (2011)

12. T.I. Shatilova, S.L. Belopukhov, E.V. Romanova, I.S. Vitol, G.P. Karpilenko, News of the TSKhA 3, 31-38 (2013)

13. J.M. Augustin, Et al., Phytochemistry 72 (6), 435-457 (2011)

14. C.L. Cantrell, F.E. Dayan, S.O. Duke, Journal of natural products 75 (6), 1231-1242 (2012)

15. O.F. Hüter, Phytochem Rev 10, 185-194 (2011) https://doi.org/10.1007/s11101-010-9168$\mathrm{y}$.

16. B. Kim, J.W. Han, M. Thi Ngo, et al., Sci Rep. 8, 14522 (2018) https://doi.org/10.1038/s41598-018-32956-4.

17. P. Parihar, S. Singh, R. Singh, V.P. Singh, S.M. Prasad, Environ. Sci. Pollut. R. 22 (6), 4056-4075 (2015)

18. N. Suzuki, R.M. Rivero, V. Shulaev, E. Blumwald, R. Mittler, New Phytol, 203, 32-43 (2014)

19. Y. Teshima, et al., Journal of agricultural and food chemistry 61 (31), 7440-7445 (2013) 